\title{
Modeling Electrokinetic Flows by Consistent Implicit Incompressible Smoothed Particle Hydrodynamics
}

\author{
Wenxiao Pan ${ }^{\mathrm{a}, *}$, Kyungjoo Kim ${ }^{\mathrm{b}}$, Mauro Perego ${ }^{\mathrm{b}}$, Alexandre M. Tartakovsky ${ }^{\mathrm{c}}$, \\ Michael L. Parks ${ }^{\mathrm{b}}$ \\ ${ }^{a}$ Department of Mechanical Engineering, University of Wisconsin-Madison, Madison, MI \\ 53706, USA \\ ${ }^{b}$ Center for Computing Research, Sandia National Laboratories, USA \\ ${ }^{c}$ Advanced Computing, Mathematics, \& Data Division, Pacific Northwest National \\ Laboratory, Richland, WA 99352, USA
}

\begin{abstract}
We present a consistent implicit incompressible smoothed particle hydrodynamics $\left(\mathrm{I}^{2} \mathrm{SPH}\right)$ discretization of Navier-Stokes, Poisson-Boltzmann, and advectiondiffusion equations subject to Dirichlet or Robin boundary conditions. It is applied to model various two and three dimensional electrokinetic flows in simple or complex geometries. The accuracy and convergence of the consistent $\mathrm{I}^{2} \mathrm{SPH}$ are examined via comparison with analytical solutions, grid-based numerical solutions, or empirical models. The new method provides a framework to explore broader applications of SPH in microfluidics and complex fluids with charged objects, such as colloids and biomolecules, in arbitrary complex geometries.

Keywords: smoothed particle hydrodynamics, electrokinetic flow, boundary condition, implicit scheme
\end{abstract}

\section{Introduction}

Smoothed particle hydrodynamics (SPH) is a Lagrangian particle-based method for solving partial differential equations (PDEs) describing momentum, mass, and energy conservation laws [1]. In Lagrangian particle-based methods, advection is modeled exactly by advecting degrees of freedom with the streamlines of

\footnotetext{
* Corresponding author

Email address: wpan9@wisc.edu (Wenxiao Pan)
}

Preprint submitted to Elsevier

October 13, 2016

(C) 2016. This manuscript version is made available under the Elsevier user license http://www.elsevier.com/open-access/userlicense/1.0/ 
the underlying flow. For flows with free surfaces and moving boundaries between different phases, the Lagrangian framework allows modeling interfaces without using (computationally expensive) front-tracking schemes. Most methods using Lagrangian moving mesh discretization are limited to flows with simple interface dynamics or small boundary movement because of the prohibitive cost associated with maintaining a high-quality mesh, as well as poor scalability. Alternatively, SPH discretizes the underlying PDEs on an assembled list of nearby particles to calculate compact interaction forces, making it more efficient and scalable. As a result, SPH has been successfully employed to model a variety of physical problems [2, including multiphase flows [3, 4, 5, 6, complex fluids [7, 8, 9], material processing [10, reactive transport in porous media [1], biomolecular diffusion [12], and proton transport in membranes [13].

Most previous SPH studies were based on the weakly compressible SPH (WCSPH) formulation that uses empirical equation of state relating the pressure to density [2]. Both, the spatial discretization and equation of state introduce inconsistencies in the numerical solution, i.e., less than second-order convergence that only can be recovered by taking increasingly large number of neighbors or an increasingly stiff equation of state [14, 15, 16]. To overcome these challenges in WCSPH, we use a consistent incompressible SPH formulation [17. Specifically, local correction matrices are computed for each particle [16] to ensure second-order discretization of differential operators. The incompressibility is enforced by solving an auxiliary pressure Poisson equation (PPE) to project the velocity into a divergence-free field [18].

The standard divergence-free incompressible SPH (ISPH) exhibits instability when particle distribution becomes highly distorted [18, 19]. This instability triggered by anisotropic particle spacing can be overcome by the enforcement of density invariance in PPE [20]. Alternatively, Ellero et al. [21] introduced the SHAKE algorithm of molecular dynamics to constrain a constant volume of every fluid particle. However, without enforcing the divergence-free condition the accuracy of prediction was deteriorated 22 . Combining divergence-free velocity and invariant density, Pozorski and Wawrenczuk suggested to solve simultane- 
ously two PPEs related to density variation and velocity divergence. Based on that, $\mathrm{Hu}$ and Adams [23] introduced internal iterations to satisfy both conditions accurately at the same moment. This method requires a higher computational cost due to many internal iterations and solving two Poisson equations at each time step. Asai et al. [24, 25] reformulated the source term of PPE to contain both conditions along with a relaxation coefficient. By such, only one PPE per time step needs to be solved. However, the relaxation coefficient must be predetermined through the hydrostatic pressure on the initial particle positions. Apart from modifying the PPE, approaches of changing particle positions were also developed in ISPH to maintain a uniform distribution of particles. For example, Chaniotis et al. 26] redistributed particles whose spacing was distorted by the flow on a uniform grid. Ngo-Cong et al. 27] solved the PPE on a set of moving integrated radial basis function network (MIRBFN) nodal points and then transferred the solution to SPH particles via interpolation. By such, the attraction of a Lagrangian method was partially lost. In the present work, we follow the approach of Xu et al. 22] and shift particles slightly across streamlines accompanied with a second-order interpolation for hydrodynamic variables at new positions. With that, particle-spacing distortion and the resulting error and instability in divergence-free ISPH are avoided without sacrificing efficiency and Lagrangian nature.

Furthermore, to overcome the time-step limitation of using explicit time integration in simulating low Reynolds number flows with high viscous dissipation, the resulting consistent incompressible SPH equations are integrated implicitly in time. In the past, implicit schemes have been introduced in SPH. In particular, Litvinov et al. 28, developed a splitting integration scheme in mesoscopic WCSPH simulations, which was considered an extension of the scheme proposed by Monaghan [29] for modeling dust-gas flow using SPH. Fan et al. [30] and Han et al. 31] updated the viscous term implicitly using iterative solvers to model non-Newtonian flows by WCSPH. Liedekerke et al. 32] employed implicit time integration in WCSPH for solving the Stokes equation without inertial terms. In the context of ISPH, Ihmsen et al. [33] used the implicit scheme to com- 
pute pressure by iteratively solving the discretized form of the PPE built from a discretized form of the continuity equation.

In this work, we propose the scalable second-order in space, implicit in time incompressible SPH ( $\left.{ }^{2} \mathrm{SPH}\right)$ discretization of fully coupled nonlinear PoissonBoltzmann (PB), Navier-Stokes (NS), and advection-diffusion (AD) equations subject to appropriate Dirichlet, Neumann, and Robin boundary conditions. Recently, I ${ }^{2} \mathrm{SPH}$ has been used to solve NS equations subject to Dirichlet boundary condition [17]. The novelty of the proposed method lies in the consistent second-order discretization and implicit time integration of the fully coupled $\mathrm{PB}$, NS, and AD equations, and imposing Robin boundary conditions using the continuous boundary force $(\mathrm{CBF})$ method 34 . As the second-order accuracy of spatial discretization is enforced at all times, to achieve the same accuracy the resulting discretization allows for a smaller compact support for neighbor particles compared with WCSPH and ISPH, leading to a smaller bandwidth of the system matrix, reducing communication between processors, and improving scalability. Moreover, the formulation allows for much larger time steps because of the improved stability by the implicit solution of the equations.

Electrokinetic flows described by coupled PB, NS, and AD equations are relevant to a variety of application areas, including microfluidics, biotechnological remediation and electrochemical technologies [35, 36, 37, 38]. In the past, incompressible SPH was used to solve coupled PB and NS equations for twodimensional (2D) droplet and Rayleigh-Taylor instability influenced by electric fields [39, 40]. The second-order accuracy of spatial discretization was not enforced in those work, and the resulting numerical errors were not reported. Here, we model electrokinetic flow in three spatial dimensions using the consistent second-order $\mathrm{I}^{2} \mathrm{SPH}$ approach and numerically demonstrate its second-order accuracy for the coupled PB and NS equations.

We use $\mathrm{I}^{2} \mathrm{SPH}$ to model electroosmotic flows in a channel, on anisotropic superhydrophobic surfaces, through a 2D charged membrane and three-dimensional (3D) charged porous media, as well as electrophoresis of a 3D spherical colloid. The method's accuracy is tested via comparison with analytical and finite ele- 
ment solutions and empirical relationships. Finally, we use the developed $\mathrm{I}^{2} \mathrm{SPH}$ method to design a microfluidic device for efficient solute transport.

\section{Governing Equations and Boundary Conditions}

We consider electrokinetic flow in a physical space $\Omega=\Omega_{f} \cup \Omega_{s}$, where fluid occupies the sub-domain $\Omega_{f}$ and $\Omega_{s}$ is occupied by solid. The boundary $\Gamma$ separates $\Omega_{f}$ and $\Omega_{s}$, i.e., $\Gamma=\Omega_{f} \cap \Omega_{s}$.

\subsection{Poisson-Boltzmann Equation}

For a system with two monovalent ions, the ions concentration $c^{+}$and $c^{-}$ and electrostatic potential $\psi$ can be determined by the $\mathrm{PB}$ equation:

$$
\nabla^{2} \tilde{\psi}=\kappa^{2} \sinh (\tilde{\psi}) \quad \text { for } \quad \mathbf{x} \in \Omega_{f}
$$

with $\kappa^{2}=\frac{2 c_{b} z^{2} e^{2}}{\epsilon_{0} \epsilon_{r} k_{B} T}$ and $\tilde{\psi}=\frac{e z}{k_{B} T} \psi$. Here, $c_{b}$ is the bulk concentration, $e$ is the elementary charge, and $z$ is the valence of ions and $z=1$ for monovalent ions. $\epsilon=\epsilon_{0} \epsilon_{r}$ denotes the absolute dielectric constant with $\epsilon_{0}$ the vacuum dielectric constant and $\epsilon_{r}$ the relative dielectric constant. $k_{B}$ is Boltzmann's constant, and $T=300 \mathrm{~K}$ is the temperature. The Debye length is defined as $\lambda_{D}=\kappa^{-1}$. The concentrations of the two ions then can be calculated as:

$$
c^{+}=e^{-\tilde{\psi}}
$$

and

$$
c^{-}=e^{\tilde{\psi}},
$$

respectively.

\subsection{Modified Poisson-Boltzmann Equation}

The modified Poisson-Boltzmann (MPB) [41] or so-called "Poisson-Fermi" equation [42] accounts for the ion size effect that becomes pronounced at high charge voltages. Hence, it presumes a different formulation for a system with two monovalent ions as:

$$
\nabla^{2} \tilde{\psi}=\kappa^{2} \frac{\sinh (\tilde{\psi})}{1+2 \gamma \sinh ^{2}(\tilde{\psi} / 2)} \quad \text { for } \quad \mathbf{x} \in \Omega_{f}
$$


Here, $\gamma \in[0,1]$ is the ratio of the total number of cations and anions in the bulk to the total number of sites available for them. The concentrations of cations and anions are then calculated as:

$$
c^{+}=\frac{e^{-\tilde{\psi}}}{1-\gamma+\gamma \cosh (\tilde{\psi})},
$$

and

$$
c^{-}=\frac{e^{\tilde{\psi}}}{1-\gamma+\gamma \cosh (\tilde{\psi})},
$$

respectively.

Given the fixed surface potential on the boundary, the PB and MPB equations satisfy the following Dirichlet boundary condition:

$$
\psi=\psi_{0} \quad \text { for } \quad \mathbf{x} \in \Gamma \text {. }
$$

\subsection{Equation for Applied Electric Field}

Following the assumption made by Henry [43] that the applied field may be simply superimposed on the electrostatic field due to electric double layer (EDL), it is computed separately when the EDL effect is absent. Assuming there are no concentration gradients in the ions that carry the current, we can express the current balance with Ohm's law and the balance equation for current density as

$$
\nabla \cdot(\sigma \nabla \phi)=0 \quad \text { for } \quad \mathbf{x} \in \Omega_{f},
$$

where, $\sigma$ denotes conductivity. At the interface with the solid, the electric potential $\phi$ and normal component of the electric displacement field $-\sigma \nabla \phi$ are continuous across the interface:

$$
\mathbf{n} \cdot\left(\sigma_{f} \nabla \phi\right)=\mathbf{n} \cdot\left(\sigma_{s} \nabla \phi\right) \quad \text { for } \quad \mathbf{x} \in \Gamma,
$$

where, $\mathbf{n}$ is the outer unit normal vector to $\Gamma$. The conductivity $\sigma$ is equal to $\sigma_{f}$ in $\Omega_{f}$ and to $\sigma_{s}$ in $\Omega_{s}$. If the solid is an insulator $\left(\sigma_{s}=0\right)$, Eq. 9 reduces to the homogeneous Neumann boundary condition:

$$
\mathbf{n} \cdot\left(\sigma_{f} \nabla \phi\right)=0 \quad \text { for } \quad \mathbf{x} \in \Gamma .
$$


Whereas, if the solid is a resistive material, Eq. (8) also is valid in the solid, and we instead can solve

$$
\nabla \cdot(\sigma \nabla \phi)=0 \quad \text { for } \quad \mathbf{x} \in \Omega=\Omega_{f} \cup \Omega_{s},
$$

which implies Eq. (9) at the interface $\Gamma$.

In addition to Eq. (9), the applied electric field is subject to the far-field boundary condition:

$$
\nabla \phi=-E_{\infty} \mathbf{e}_{\mathbf{x}} \quad \text { for } \quad \mathbf{x} \rightarrow \infty
$$

where $E_{\infty}$ is the external electric field and $\mathbf{e}_{\mathbf{x}}$ is the unit vector in the direction of the $x$-axis. Because the computational domain has to be finite, the far-field condition should be properly replaced. In practice, buffer zones (mimicking the electrodes positioned to generate the electric field) are attached to the fluid domain with extrapolated electric potentials according to Eq. (11). As such, the far-field condition is replaced with the Dirichlet boundary conditions.

\subsection{Navier-Stokes Equations}

The external electric field $E_{\infty}$ exerts the electrostatic body force $\mathbf{f}_{E}$ on the fluid and may cause advection of ions and charged surfaces. The fluid velocity $\mathbf{v}$ is assumed to satisfy the incompressible NS equations:

$$
\left\{\begin{array}{l}
\frac{d \mathbf{v}}{d t}=-\frac{\nabla P}{\rho}+\nabla \cdot(\nu \nabla \mathbf{v})+\mathbf{g}+\frac{\mathbf{f}_{E}}{\rho} \\
\nabla \cdot \mathbf{v}=0 \quad \text { for } \quad \mathbf{x} \in \Omega_{f},
\end{array}\right.
$$

where, $\mathbf{g}$ is the gravitational body force per unit mass acting on the fluid, such as gravity, $\nu=\frac{\mu}{\rho}$ is the kinematic viscosity of the fluid, and $\rho$ and $\mu$ are fluid density and viscosity, respectively. The electrostatic force is given by

$$
\mathbf{f}_{E}=-c_{b} \sum_{k=1}^{N} e z^{k} c^{k}(\nabla \psi+\nabla \phi),
$$

where, $c^{k}$ is the normalized ion concentration of species $k$. For a two-ion monovalent system $\left(\left|z^{k}\right|=1\right), \mathbf{f}_{E}$ can be simplified as:

$$
\mathbf{f}_{E}=-e c_{b}\left(c^{+}-c^{-}\right)(\nabla \psi+\nabla \phi) .
$$


In general, at the fluid-solid boundary, the fluid velocity can be different from the boundary velocity. The slip length magnitude depends on the fluid's wetting properties and surface roughness. On the macroscale, the slip length is much smaller than the characteristic domain size, and the no-slip boundary condition for the NS equation is commonly used:

$$
\mathbf{v}=\mathbf{v}_{\Gamma} \quad \text { for } \quad \mathbf{x} \in \Gamma
$$

where $\mathbf{v}_{\Gamma}$ is the velocity of the boundary $\Gamma$. On the microscale, the effect of a non-zero slip on the overall flow may be significant, and the stress-dependent velocity could be prescribed via the Navier boundary condition [44]:

$$
(\boldsymbol{\tau} \cdot \mathbf{n})_{\|}=k \tilde{\mathbf{v}}_{\|} \quad \text { for } \quad \mathbf{x} \in \Gamma
$$

where $\boldsymbol{\tau}(\mathbf{x}, t)=\mu\left\{\nabla \mathbf{v}(\mathbf{x}, t)+[\nabla \mathbf{v}(\mathbf{x}, t)]^{\mathrm{T}}\right\}$ is the deviatoric stress tensor; $\tilde{\mathbf{v}}=$ $\mathbf{v}-\mathbf{v}_{\Gamma} ; k$ is the friction coefficient, a property of the solid boundary; "||" denotes the tangential component of a vector to the boundary. Here, we treat the boundary as impermeable, setting the normal velocity at the boundary as:

$$
\mathbf{v} \cdot \mathbf{n}=\mathbf{v}_{\Gamma} \cdot \mathbf{n} \quad \text { for } \quad \mathbf{x} \in \Gamma .
$$

The friction coefficient is assumed to be constant in the Navier boundary condition, and it is related to the slip length $L_{s}$ as $k=\frac{\mu}{L_{s}}$. The slip length is equal to the distance normal to the boundary at which the extrapolated velocity profile reaches zero. The Navier boundary condition has been shown both experimentally and numerically to be valid only at isotropic surfaces and in low shear rate limits [45, 46, 47]. Under more general conditions, the Navier boundary condition should be replaced with a more general Robin boundary condition:

$$
(\boldsymbol{\tau} \cdot \mathbf{n})_{\|}=\mathbf{f}_{\Gamma}\left(\mathbf{x}, \tilde{\mathbf{v}}_{\|}\right) \quad \text { for } \quad \mathbf{x} \in \Gamma \text {. }
$$

To make the NS equation with Robin boundary condition amenable to discretization with SPH, we approximate it using the CBF method [34. The main idea of the CBF method is to replace the Robin boundary condition (Eq. (18) ) 
with a homogeneous Neumann boundary condition and a corresponding volumetric source term, $\mathbf{f}_{\Omega}\left(\mathbf{x}, \tilde{\mathbf{v}}_{\|}\right)$, added into the momentum equation (Eq. 12 ). Thus, we approximate Eqs. (12) and (18) with

$$
\left\{\begin{array}{l}
\frac{d \mathbf{v}^{r}}{d t}=-\frac{\nabla P^{r}}{\rho}+\nabla \cdot\left(\nu \nabla \mathbf{v}^{r}\right)+\mathbf{g}+\frac{\mathbf{f}_{E}}{\rho}-\frac{\mathbf{f}_{\Omega}}{\rho} \\
\nabla \cdot \mathbf{v}^{r}=0 \quad \text { for } \quad \mathbf{x} \in \Omega_{f},
\end{array}\right.
$$

subject to the homogeneous Neumann boundary condition:

$$
\left(\boldsymbol{\tau}^{r} \cdot \mathbf{n}\right)_{\|}=0, \quad \text { for } \quad \mathbf{x} \in \Gamma
$$

where

$\mathbf{f}_{\Omega}\left(\mathbf{x}, \tilde{\mathbf{v}}_{\|}^{r}\right)=\mathbf{f}_{\Gamma}\left(\mathbf{x}, \tilde{\mathbf{v}}_{\|}^{r}\right) \iint_{\Omega_{s}}\left[\mathbf{n}(\mathbf{x})+\mathbf{n}\left(\mathbf{x}^{\prime}\right)\right] \cdot \nabla_{\mathbf{x}} W\left(\mathbf{x}-\mathbf{x}^{\prime}, h_{r}\right) d \mathbf{x}^{\prime} \quad$ for $\quad \mathbf{x} \in \Omega_{f}$

Here, the normalized kernel function, $W$, is a positive bell-shaped function with, at least, first continuous derivative and compact support $\omega h_{r}\left(W\left(|\mathbf{r}|>\omega h_{r}\right)=\right.$ $0)$. The value of $\omega$ depends on the choice of $W$. In addition, $W$ satisfies the following conditions:

$$
\iiint_{\Omega_{f} \cup \Omega_{s}} W\left(\mathbf{x}-\mathbf{x}^{\prime}, h_{r}\right) d \mathbf{x}^{\prime}=1
$$

and

$$
\lim _{h_{r} \rightarrow 0} W\left(\mathbf{x}-\mathbf{x}^{\prime}, h_{r}\right)=\delta\left(\mathbf{x}-\mathbf{x}^{\prime}\right) .
$$

In 34, it has been shown that:

$$
\lim _{h_{r} \rightarrow 0} \mathbf{v}^{r}=\mathbf{v} \text { and } \quad \lim _{h_{r} \rightarrow 0} P^{r}=P .
$$

To simplify notation, we omit the superscript $r$ in subsequent derivations for variables in the $\mathrm{CBF}$ equations.

\subsection{Advection-Diffusion Equation}

The transport of dissolved substances under electrokinetic flows can be described by the $\mathrm{AD}$ equation:

$$
\frac{d S}{d t}=\frac{\partial S}{\partial t}+(\mathbf{v} \cdot \nabla) S=\nabla \cdot(D \nabla S) \quad \text { for } \quad \mathbf{x} \in \Omega_{f},
$$


where $S$ is the normalized concentration of a solute, $\frac{d S}{d t}$ is the material derivative of $S$, and $D$ is the diffusion coefficient. Assuming constant input concentration at the inlet boundary $\Gamma_{\text {in }}$ and the absence of surface reactions on $\Gamma$, Eq. 25 is subject to the boundary condition:

$$
\begin{cases}S=S_{0}\left(\mathbf{x}_{0}\right) & \mathbf{x} \in \Gamma_{\text {in }} \\ \mathbf{n} \cdot(D \nabla S)=0 & \mathbf{x} \in \Gamma .\end{cases}
$$

\section{3. $\mathrm{I}^{2} \mathrm{SPH}$ method}

\subsection{Spatial Discretization}

In SPH, the domains $\Omega_{f}$ and $\Omega_{s}$ are discretized with two sets of particles. The particles within $\Omega_{f}$ and $\Omega_{s}$ are referred to as "fluid" and "solid" particles, respectively. Then, the point values $\left\{\mathbf{v}_{i}, P_{i}, \rho_{i}, \nu_{i}, \phi_{i}, \psi_{i}, S_{i}\right\}$ are associated with $\mathbf{x}_{i}$, the position of particle $i$.

The SPH discretization is based on a meshless interpolation scheme:

$$
A_{i} \approx \sum_{j} A_{j} W_{i j} V_{j}
$$

where $A_{i}=A\left(\mathbf{x}_{i}\right)$ is a function defined at point $i, W_{i j}=W\left(\mathbf{r}_{i j}\right), r_{i j}=\left|\mathbf{r}_{i j}\right|$, and $\mathbf{r}_{i j}=\mathbf{x}_{i}-\mathbf{x}_{j} . V_{i}$ denotes the particle volume,

$$
V_{i}=\left(\sum_{j \in N_{\epsilon, i}} W_{i j}\right)^{-1},
$$

where $W$ is the weighting function defined in Section 2.4. In the present work, we use the quintic Wendland kernel that has continuous and smooth first and second derivatives and is defined as:

$$
W(\mathbf{r}, h)=\frac{\alpha_{d}}{h^{d}}(1-0.5 q)^{4}(2 q+1) \quad 0 \leq q \leq 2,
$$

where $q=r / h, d$ is the spatial dimension $(d=2,3)$ and $\alpha_{d}$ is a normalization constant $\left(\alpha_{d}=7 / 4 \pi\right.$ for $d=2$ and $21 / 16 \pi$ for $\left.d=3\right)$. With this form of $W$, only neighbor particles of particle $i$ in the set $N_{h, i}=\left\{\mathbf{x}_{j}\right.$ s.t. $\left.\left|\mathbf{x}_{j}-\mathbf{x}_{i}\right|<2 h\right\}$ contribute to the summation in the interpolation scheme (Eq. 27]). 
In $\mathrm{I}^{2} \mathrm{SPH}, \nabla \cdot(a \nabla A)$ is decomposed as:

$$
\nabla \cdot(a \nabla A)=a \nabla^{2} A+(\nabla a) \cdot(\nabla A)
$$

for discretization. Meanwhile, the differential operators $\nabla$ and $\nabla^{2}$ are discretized using "consistent" schemes as:

$$
\nabla_{h} A_{i}=\sum_{j \in N_{h, i}}\left(A_{j}-A_{i}\right) \mathbf{G}_{i} \nabla_{i} W_{i j} V_{j}
$$

and

$$
\nabla_{h}^{2} A_{i}=2 \sum_{j \in N_{h, i}}\left(\mathbf{L}_{i}: \mathbf{e}_{i j} \otimes \nabla_{i} W_{i j}\right)\left(\frac{A_{i}-A_{j}}{r_{i j}}-\mathbf{e}_{i j} \cdot \nabla_{h} A_{i}\right) V_{j},
$$

where $\mathbf{e}_{i j}=\mathbf{r}_{i j} / r_{i j}$. The correction tensors $\mathbf{G}_{i}$ and $\mathbf{L}_{i}$ guarantee exact reproduction of the gradient for linear functions and the Laplacian for parabolic functions, respectively. They are specifically given as [16]:

$$
\mathbf{G}_{i}=-\left[\sum_{j \in N_{h, i}} \mathbf{r}_{i j} \nabla_{i} W_{i j} V_{j}\right]^{-1}
$$

and

$\mathbf{L}_{i}:\left[\sum_{j \in N_{h, i}} \mathbf{r}_{i j} \mathbf{e}_{i j} \mathbf{e}_{i j} \nabla_{i} W_{i j} V_{j}+\left(\sum_{j \in N_{h, i}} \mathbf{e}_{i j} \mathbf{e}_{i j} \nabla_{i} W_{i j} V_{j}\right) \cdot \mathbf{G}_{i} \cdot\left(\sum_{j \in N_{h, i}} \mathbf{r}_{i j} \mathbf{r}_{i j} \nabla_{i} W_{i j} V_{j}\right)\right]=-\mathbf{I}$.

The correction tensors' construction is performed locally, i.e., $\mathbf{G}_{i}$ and $\mathbf{L}_{i}$ are obtained by inversion of $3 \times 3$ and $6 \times 6$ matrices, respectively, with components depending on the particle positions within $N_{h, i}$. The particles within $N_{h, i}$ can be easily found using a linked list without the need for a global search over all particles. Because of this, calculations of $\mathbf{G}_{i}$ and $\mathbf{L}_{i}$ do not require additional interprocessor communications [17. This discretization of spatial derivatives achieves a consistent second-order convergence with respect to $h$, which does not depend on the ratio of $\Delta x / h(\Delta x$ : the initial particle spacing on the Cartesian grid) as in the classical SPH discretization [16]. Therefore, $\mathrm{I}^{2} \mathrm{SPH}$ allows for a smaller compact support of neighbor particles and has higher accuracy and reduced operation count compared to inconsistent SPH approaches. In fact, the results presented in this work utilize a short smoothing length $h=1.0 \Delta x$. 
From Eqs. (30) and (31), we note that linear and angular momenta are not exactly conserved in the consistent $\mathrm{I}^{2} \mathrm{SPH}$ discretization but with the secondorder numerical error. As discussed in [48, 49, a nonconservative SPH scheme may lead to unphysical errors for problems where the conservation of linear and angular momenta is essential. For those problems, the quality of conservative inconsistent SPH approximation relies on the conservative properties of the Hamiltonian formulation to achieve useful results rather than attempting to achieve convergence to the exact solution of PDEs [50]. However, for studying the low Reynolds number electrokinetic flows as in the present work, the physics is dominated by the second-order operator in the viscous term of NS equation and the Laplacian of potential in the PB equation. Thus, due to the dissipative property of the second-order operator, accuracy and convergence to the exact solution of governing PDEs is more critical. Further, numerical experiments [15, 51] and some analytic estimates [16] have demonstrated that the secondorder operator is more sensitive to particle anisotropy, and the inconsistent SPH discretization diverges as the number of particles used in the domain increases.

\subsection{Boundary Conditions}

The SPH approximations of fields and their spatial derivatives require full support of the kernel contained in the domain $\left(\forall i, \operatorname{supp}\left(W\left(\left|x-x_{i}\right|\right) \subseteq \Omega_{f}\right)\right.$. For particles near the boundary, the truncation is remedied by introducing several layers of ghost (fixed dummy) particles in the solid (or buffer zone) and performing a linear extrapolation of the state variables to the ghost particles [52, 53].

Essential boundary conditions. Consider a fluid particle $f$ and a solid particle $s$. For a Dirichlet boundary condition, the function $A$ is extended as:

$$
A_{s}=\frac{d_{s}}{d_{f}}\left(A_{\Gamma}-A_{f}\right)+A_{\Gamma},
$$

where $d_{s}$ and $d_{f}$ denote closest perpendicular distances to the boundary for the solid and fluid particles, respectively. Here, $A_{\Gamma}$ is the Dirichlet data on 
the boundary. For a fluid particle $i$ and a solid particle $j$, the term $\left(A_{j}-A_{i}\right)$

of the sum in (30) and (31) is given by $\left(1+\frac{d_{j}}{d_{i}}\right)\left(A_{\Gamma}-A_{i}\right)$. As discussed in the continuum limit by Macia et al. [54, applying the linear extrapolation introduces a local $\mathcal{O}\left(h^{2}\right)$ error that matches the second-order accuracy of the consistent $\mathrm{I}^{2} \mathrm{SPH}$.

Computing $d_{i}$ and $d_{j}$ for general geometries would require a spline representation of the boundary, reintroducing a mesh into the problem. In practice, these distances can be approximated using a smoothed approximation [55]. Specifically, an indicator function is defined to differentiate fluid and solid particles as:

$$
\chi_{f}=\frac{\sum_{j \in \text { fluid }} W\left(r_{f j}\right)}{\sum_{j} W\left(r_{f j}\right)}, \quad \chi_{s}=\frac{\sum_{j \in \text { solid }} W\left(r_{s j}\right)}{\sum_{j} W\left(r_{s j}\right)} .
$$

Thereby, the distance to the boundary can be approximated for either fluid or solid particles using

$$
d_{i}=\omega h\left(2 \chi_{i}-1\right)
$$

where $\omega h$ is the kernel compact support with $\omega=2$ for the Wendland kernel function (Eq. 29) used in this work. In practice, this allows the specification of an arbitrarily complex geometry by simply placing a lattice of particles over a domain $\Omega$ and marking particles as either fluid or solid. For many applications, this framework allows trivial discretization of experimentally available datasets (e.g., geometry specified from voxel data from magnetic resonance imaging (MRI)).

Dirichlet boundary conditions for a vector field such as $\mathbf{v}$ are prescribed analogously by substituting, component-wise, $\left(\mathbf{v}_{j}-\mathbf{v}_{i}\right)$ with

$$
\left(1+\frac{d_{j}}{d_{i}}\right)\left(\mathbf{v}_{\Gamma}-\mathbf{v}_{i}\right)
$$

in Eqs. (30) and (31) for fluid particles $i$ and solid particle $j$. If we want to prescribe a Dirichlet condition only along a direction represented by the unit vector $\mathbf{n}_{i}$, then the term $\left(\mathbf{v}_{j}-\mathbf{v}_{i}\right)$ must be replaced with

$$
\left(1+\frac{d_{j}}{d_{i}}\right)\left(\mathbf{v}_{\Gamma} \cdot \mathbf{n}_{i}-\mathbf{v}_{i} \cdot \mathbf{n}_{i}\right) \mathbf{n}_{i}
$$


In case of the no-penetration boundary condition, $\mathbf{n}_{i}$ is the normal unit vector at fluid particle $i$ to the boundary and can be computed, as explained in the following, using expression Eq. (37).

Natural boundary conditions. The homogeneous Neumann boundary condition is enforced by including only the fluid particles in the summation, which is equivalent to $A_{s}=A_{f}$, as commonly employed in SPH models for free-surface flow [56].

To impose the Robin boundary condition, the volumetric term (Eq. (21)) in Eq. (19) is discretized as:

$$
\begin{aligned}
\mathbf{f}_{\Omega}\left(\mathbf{x}, \tilde{\mathbf{v}}_{\|}\right) & =\mathbf{f}_{\Gamma}\left(\mathbf{x}, \tilde{\mathbf{v}}_{\|}\right) \iiint_{\Omega_{s}}\left[\mathbf{n}(\mathbf{x})+\mathbf{n}\left(\mathbf{x}^{\prime}\right)\right] \cdot \nabla_{\mathbf{x}} W\left(\mathbf{x}-\mathbf{x}^{\prime}\right) d \mathbf{x}^{\prime} \\
& =\mathbf{f}_{\Gamma}\left(\mathbf{x}, \tilde{\mathbf{v}}_{\|}\right) \sum_{j \in \text { solid }}\left(\mathbf{n}(\mathbf{x})+\mathbf{n}_{j}\right) \cdot \nabla_{\mathbf{x}} W\left(\mathbf{x}-\mathbf{x}_{j}\right) V_{j},
\end{aligned}
$$

where $\sum_{j \in \text { solid }}$ is the summation over solid particles in the neighbor set $N_{h, i}$. Evaluating Eq. 35 at $\mathbf{x}_{i}$ yields:

$$
\mathbf{f}_{\Omega, i}=\mathbf{f}_{\Gamma}\left(\mathbf{x}_{i}, \tilde{\mathbf{v}}_{\|, i}\right) \sum_{j \in \text { solid }}\left(\mathbf{n}_{i}+\mathbf{n}_{j}\right) \cdot \nabla_{x_{i}} W_{i j} V_{j} .
$$

The normal unit vector $\mathbf{n}_{i}$ is calculated as:

$$
\mathbf{n}_{i}=\frac{\sum_{j}\left(\Theta_{j}-\Theta_{i}\right) \nabla_{x_{i}} W_{i j} V_{j}}{\left|\sum_{j}\left(\Theta_{j}-\Theta_{i}\right) \nabla_{x_{i}} W_{i j} V_{j}\right|},
$$

where the color function $\Theta$ is equal to zero for fluid particles and one for solid particles [34.

\subsection{Implicit Scheme}

For solving the incompressible NS equation, the predictor-corrector [18] with second-order incremental projection scheme [17] is used. We first solve a Helmholtz equation for each fluid particle $i$ to obtain an intermediate velocity. Assuming the no-slip boundary condition, we have

$$
\begin{cases}\frac{\mathbf{v}_{i}^{*}-\mathbf{v}_{i}^{n}}{\Delta t}=-\frac{1}{\rho_{i}} \nabla P_{i}^{n}+\nabla \cdot\left[\frac{\nu}{2} \nabla\left(\mathbf{v}_{i}^{*}+\mathbf{v}_{i}^{n}\right)\right]+\mathbf{g}+\frac{\mathbf{f}_{E, i}}{\rho_{i}} & \mathbf{x}_{i} \in \Omega_{f} \\ \mathbf{v}_{i}^{*}=\mathbf{v}_{\Gamma} & \mathbf{x}_{i} \in \Gamma .\end{cases}
$$


For the Robin boundary condition, the Helmholtz equation becomes

$$
\begin{cases}\frac{\mathbf{v}_{i}^{*}-\mathbf{v}_{i}^{n}}{\Delta t}=-\frac{1}{\rho_{i}} \nabla P_{i}^{n}+\nabla \cdot\left[\frac{\nu_{i}}{2} \nabla\left(\mathbf{v}_{i}^{*}+\mathbf{v}_{i}^{n}\right)\right]+\mathbf{g}+\frac{\mathbf{f}_{E, i}}{\rho_{i}}-\frac{\mathbf{f}_{\Omega, i}}{\rho_{i}} & \mathbf{x}_{i} \in \Omega_{f}, \\ \mathbf{v}_{i}^{*} \cdot \mathbf{n}=\mathbf{v}_{\Gamma} \cdot \mathbf{n} & \mathbf{x}_{i} \in \Gamma, \\ \partial_{n} \mathbf{v}_{\|, i}^{*}=0 & \mathbf{x}_{i} \in \Gamma .\end{cases}
$$

Substituting $\mathbf{v}_{i}^{*}$ into Eq. (36), we obtain:

$$
\mathbf{f}_{\Omega, i}=\mathbf{f}_{\Gamma}\left(\mathbf{x}_{i}, \mathbf{v}_{\|, i}^{*}-\mathbf{v}_{\Gamma, \|}\right) \sum_{j \in \text { solid }}\left(\mathbf{n}_{i}+\mathbf{n}_{j}\right) \cdot \nabla_{x_{i}} W_{i j} V_{j} .
$$

After that, a Poisson equation is solved for a pressure increment $q=P^{n+1}-P^{n}$ to make the velocity $\mathbf{v}^{n+1}=\mathbf{v}^{*}-\frac{\Delta t}{\rho} \nabla q$ divergence-free,

$$
\begin{cases}\nabla \cdot\left(\frac{1}{\rho_{i}} \nabla q_{i}\right)=\frac{\nabla \cdot \mathbf{v}_{i}^{*}}{\Delta t} & \mathbf{x}_{i} \in \Omega_{f} \\ \partial_{n} q_{i}=0 & \mathbf{x}_{i} \in \Gamma\end{cases}
$$

Finally, the pressure and positions are updated, taking into account the fact that the predictor pressure $P_{i}^{n}$ and corrected pressure $P_{i}^{n+1}$ are associated with different particle positions $\mathbf{x}_{i}^{n}$ and $\mathbf{x}_{i}^{n+1}$ :

$$
\left\{\begin{array}{l}
\mathbf{x}_{i}^{n+1}=\mathbf{x}_{i}^{n}+\Delta t \frac{\mathbf{v}_{i}^{n+1}+\mathbf{v}_{i}^{n}}{2} \\
P_{i}^{n+1}\left(\mathbf{x}_{i}^{n+1}\right)=P_{i}^{n}\left(\mathbf{x}_{i}^{n}\right)+q_{i}^{n+1}\left(\mathbf{x}_{i}^{n}\right)+\nabla P_{i}^{n+1}\left(\mathbf{x}_{i}^{n}\right) \cdot\left(\mathbf{x}_{i}^{n+1}-\mathbf{x}_{i}^{n}\right) .
\end{array}\right.
$$

For solving the $\mathrm{AD}$ equation (Eq. (25)) to describe the solute transport, particles are advected with the flow, and the time derivative is computed implicitly. Specifically, at each time step, a Poisson problem is solved to update the concentration:

$$
\begin{cases}\frac{\mathrm{S}_{i}^{n+1}-\mathrm{S}_{i}^{n}}{\Delta t}=\nabla \cdot\left[\frac{D_{i}}{2} \nabla\left(S_{i}^{n+1}+S_{i}^{n}\right)\right] & \mathbf{x}_{i} \in \Omega_{f} \\ \partial_{n} \mathrm{~S}_{i}=0 & \mathbf{x}_{i} \in \Gamma\end{cases}
$$

The associated linear equations resulting from the above implicit integration schemes (Eqs. (38)-(39), 41), and 433) are solved using the Trilinos solver libraries [57] - specifically, Belos for a GMRES linear solver [58] and ML for an algebraic multigrid (AMG) preconditioner [59]. 
Using this implicit integrator, the appropriate time step is chosen to ensure accuracy and numerical stability for the solution according to:

$$
\Delta t \leq \alpha \frac{\Delta x}{|\mathbf{v}|_{\max }},
$$

with $\alpha=0.01-0.1$. Specifically, $\alpha=0.1$ was used in those cases with a flow in a 2D channel (Sections 4.1, 4.3, and 4.6), and in the other cases $\alpha=0.01$.

\subsection{Particle Regularity}

As SPH particles advect with flow, the particles could clump together or form voids near stagnation points. Thus, the standard divergence-free ISPH exhibits instability when particle distribution becomes highly distorted [18, 19]. Here, our divergence-free approach maintains accuracy and stability without sacrificing efficiency by slightly shifting particles away from streamlines, following the work of Xu et al. 22. Specifically, an anisotropy indicator is used at the end of every time step to shift particles and enforce a uniform particle number density, which is calculated from:

$$
\delta \mathbf{r}_{i}=s|\mathbf{v}|_{\max } \Delta t \sum_{j \in N_{h, i}} \frac{\bar{r}_{i}^{2}}{r_{i j}^{2}} \mathbf{e}_{i j}, \quad \bar{r}_{i}=\frac{1}{\#\left\{j \in N_{h, i}\right\}} \sum_{j \in N_{h, i}} r_{i j},
$$

where $s$ is an adjustable coefficient. The particles' positions are updated according to:

$$
\mathbf{x}_{i}^{n+1, \text { corr }}=\mathbf{x}_{i}^{n+1}+\delta \mathbf{r}_{\mathbf{i}},
$$

and accordingly, the field variables are corrected by the Taylor series:

$$
A_{i}^{n+1, \text { corr }}=A_{i}^{n+1}+\nabla A_{i}^{n+1} \cdot \delta \mathbf{r}_{\mathbf{i}}+\mathcal{O}\left(\delta \mathbf{r}_{\mathbf{i}}^{2}\right),
$$

where $\nabla A_{i}^{n+1}$ is calculated according to Eq. 30 . We note that the particle shift is, in principle, compromising the Lagrangian nature of the discretization. However, the amount of shifting actually needed in our simulations is quite small. Hence, we actually do not loose the advantages of a Lagrangian scheme. With particle regularity well maintained and the consistent second-order discretization of differential operators, our projection-based $\mathrm{I}^{2} \mathrm{SPH}$ has the second-order spatial accuracy, matching that of finite volume methods, while keeping the attraction of a mesh-free Lagrangian method. 


\subsection{Scalable Implementation}

The 3D massively parallel code for the present $\mathrm{I}^{2} \mathrm{SPH}$ method to model electrokinetic flows has been implemented within the Large-scale Atomic/Molecular Massively Parallel Simulator (LAMMPS) framework [60, which provides software infrastructure for particle-based methods with efficient parallel algorithms. LAMMPS has multiple strategies to map processors to a problem domain. Cartesian decomposition is used for simple box geometries. For more complicated geometry, LAMMPS uses a recursive coordinate bisection approach so it can maintain nearly perfect load balance among processors. The graph of the matrix needed by Trilinos solver libraries can be easily extracted from the neighbor lists in LAMMPS with little additional overhead communications.

\section{Simulation results}

In this section, we present simulations of electrokinetic flows and examine numerical accuracy of $\mathrm{I}^{2} \mathrm{SPH}$. The numerical $\mathrm{I}^{2} \mathrm{SPH}$ solutions are compared with analytical solutions, numerical solutions obtained using the finite element method (FEM), or phenomenological results obtained from experiments. In all cases, the fluid is assumed to be an aqueous solution of two monovalent ions with density $\rho=1000 \mathrm{~kg} / \mathrm{m}^{3}$, viscosity $\mu=1 \times 10^{-3} \mathrm{~Pa} \cdot \mathrm{s}$, and relative permittivity $\epsilon_{r}=80$.

\subsection{Electroosmotic Flow in a Channel}

First, we consider an electroosmotic flow governed by the NS and PB equations in a $2 \mathrm{D}$ straight channel with a fixed electrostatic surface potential $\left(\psi_{0}\right)$. The channel is oriented along the $x$ coordinate with impermeable walls (plates) located at $y=H=20 \mathrm{~nm}$ and $y=-H=-20 \mathrm{~nm}$. The no-slip boundary condition for the fluid velocity is imposed at the two plates, and the periodic boundary conditions at the remaining boundaries. Initially, both electrostatic potential and velocity of fluid are set at zero. The Debye length is set to $\lambda_{D}=2$ $\mathrm{nm}$, and $E_{\infty}=10^{6} \mathrm{~V} / \mathrm{m}$ is set for the applied electric field. The flow is solely driven by the electrostatic force, i.e., $g=0$. 

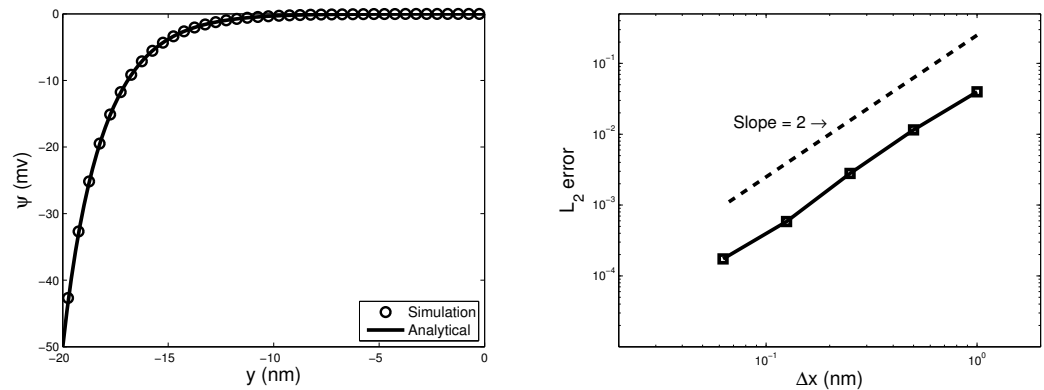

Figure 1: (Left) Profile of electrostatic potential across the channel width at spatial resolution $\Delta x=0.5 \mathrm{~nm}$, compared with the analytical solution; (right) corresponding $L_{2}$ relative errors at varying spatial resolutions.
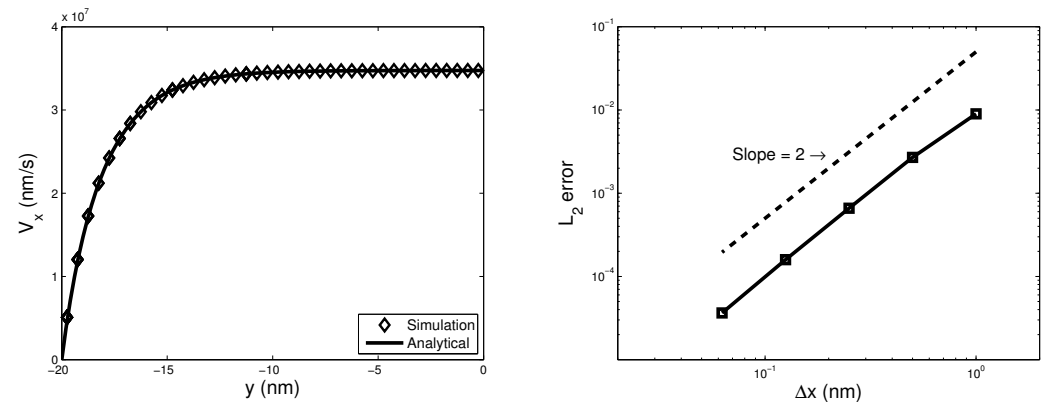

Figure 2: (Left) Steady-state profile of electroosmotic flow velocity across the channel width at spatial resolution $\Delta x=0.5 \mathrm{~nm}$, compared with the analytical solution; (right) corresponding $L_{2}$ relative errors at varying spatial resolutions.

The profile of electrostatic potential and steady-state velocity then are computed and compared with their corresponding analytical solutions. Good agreement between the $\mathrm{I}^{2} \mathrm{SPH}$ and analytical solutions with a second-order convergence is achieved for both electrostatic potential and velocity, as depicted in Figures 1 and 2, respectively. Because of symmetry, only half of the solution profiles $(-H<y \leq 0)$ are plotted.

We also investigate the effect of ion size on the electrostatic potential and electroosmotic flow velocity. To this end, for a high voltage $\left(\psi_{0}=-200 \mathrm{mV}\right)$, we solve the MPB equation (Eq. (4)) with a nonzero $\gamma$. The resulting potential profile across the channel width is far less steep than that is predicted by the 

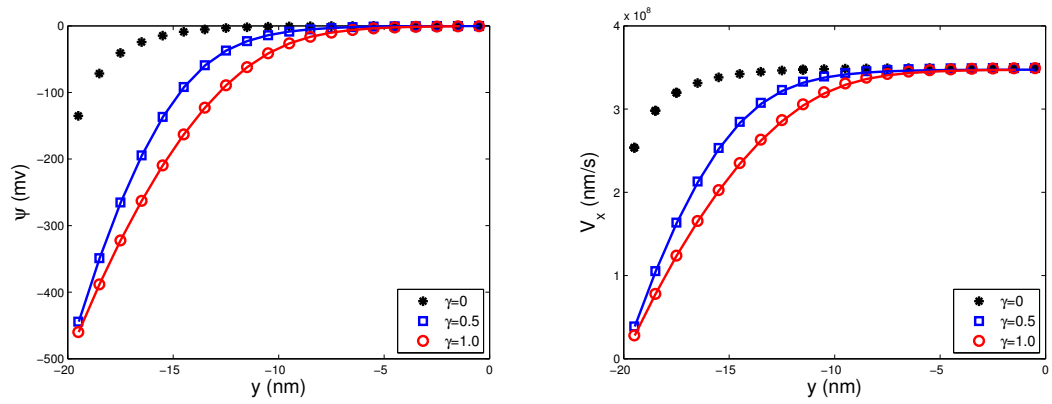

Figure 3: Effects of ion size on electrostatic potential (left) and electroosmotic flow velocity (right) at $\psi_{0}=-200 \mathrm{mV}$. Symbols and solid lines denote numerical and semi-analytical simulations, respectively.

Gouy-Chapman theory $(\gamma=0)$ due to the fact that ions cannot pack densely near the surface to screen very high surface potential. Rather, they occupy extended layers, which, in turn, changes the velocity profile and reduces the flow rate 61 as shown in Figure 3 . Here, comparison with the semi-analytical solutions of MPB with nonzero $\gamma$ [42] demonstrates the accuracy of $\mathrm{I}^{2} \mathrm{SPH}$ solutions in predicting the ion size effects.

\subsection{Electroosmotic Flow through a Charged Membrane}

Second, we examine the electroosmotic flow through a 2D $(x, y)$ charged membrane modeled by a a periodic lattice of charged uniform cylinders. A fixed potential $\psi_{0}$ and the no-slip boundary condition for the flow velocity are prescribed on the cylinders' surfaces, and the periodic boundary conditions are imposed at the external boundaries. Initially, both electrostatic potential and velocity of bulk fluid are set at zero, and the Debye length is set to $\lambda_{D}=1 \mathrm{~nm}$.

The applied electric field is computed with $E_{\infty}=10^{6} \mathrm{~V} / \mathrm{m}$ and $\sigma_{f}=\sigma_{s}$. Along with the applied electric field, a body force $\mathbf{g}=-g \mathbf{e}_{\mathbf{y}}$ is also employed to drive the flow with $g=4.0 \times 10^{11} \mathrm{~m} / \mathrm{s}^{2}$. We first examine the electrostatic potential (shown in Figure 4 ) in the bulk solvent with $\psi_{0}=-50 \mathrm{mV}$ by numerically solving the $\mathrm{PB}$ and NS equations using $\mathrm{I}^{2} \mathrm{SPH}$. The flow velocities also are computed at different times. Figures 5 and 6 show good agreement between 


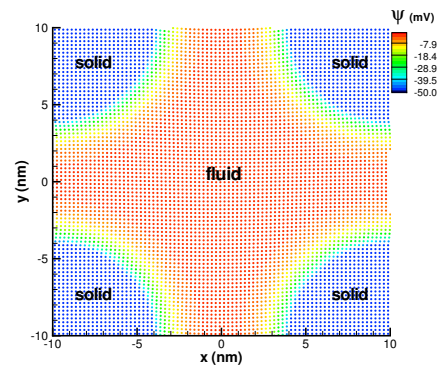

Figure 4: Contour of electrostatic potential distribution in the bulk fluid with a fixed potential $\left(\psi_{0}=-50 \mathrm{mV}\right)$ on the solid.

fluid velocities obtained from $\mathrm{I}^{2} \mathrm{SPH}$ and FEM at a higher resolution.

Furthermore, we investigate the effects of ion size at a high voltage $\left(\psi_{0}=\right.$ $-200 \mathrm{mV}$ ) in the absence of gravitational body force (i.e., $g=0$ ). Figure 7 depicts the computed electrostatic potential and contours of the $x$ velocity component at different values of $\gamma$. With an increasing $\gamma$ in the MPB equation (i.e., with the increasing effect of the ion size), the potential distribution becomes less steep near the boundary because finite-size ions extend their occupancy apart from the boundary. Hence, the resulting velocity varies less abruptly near the boundaries.

\subsection{Electroosmotic Flow on Anisotropic Superhydrophobic Surfaces}

Third, we study the electroosmotic flows on anisotropic superhydrophobic surfaces. Slip-enhanced electroosmotic flows has been actively studied in microand nano-fluidics 62. On hydrophobic surfaces with nonzero slip lengths, flows generally are subject to the slip boundary condition (Eq. (18)). To demonstrate the accuracy and convergence of the developed $\mathrm{I}^{2} \mathrm{SPH}$ method in imposing the Robin boundary condition using the CBF method [34, we consider a flow in a 2D straight channel bounded by impermeable plates at the top $(y=H=20$ $\mathrm{nm})$ and bottom $(y=-H=-20 \mathrm{~nm})$ with slip length $L_{s}=10 \mathrm{~nm}$. The flow is driven by a body force $g \mathbf{e}_{\mathbf{x}}$ with $g=10^{8} \mathrm{~m} / \mathrm{s}^{2}$. The Navier slip boundary condition (Eq. (16) ) is imposed at the two plates, and the periodic boundary 

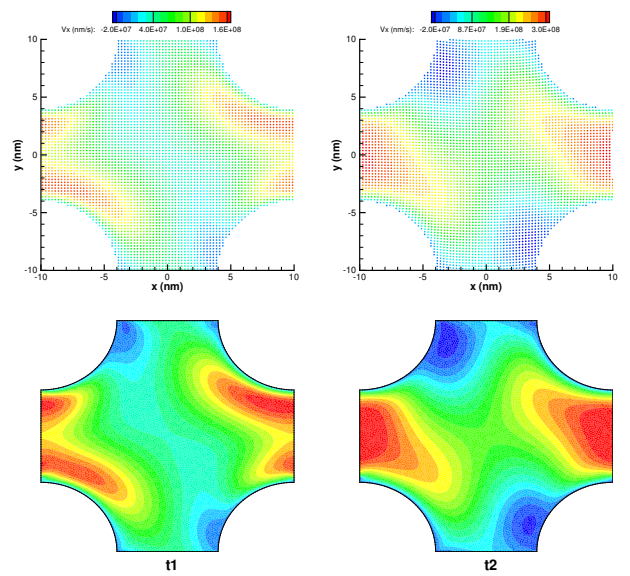

Figure 5: Contours of $v_{x}$ at times $t_{1}=0.264 \mathrm{ps}$ and $t_{2}=2.64 \mathrm{ps,} \mathrm{computed} \mathrm{by} \mathrm{I}^{2} \mathrm{SPH}$ with the spatial resolution $\Delta x=0.25 \mathrm{~nm}$ (top) and FEM with $\Delta x=0.2 \mathrm{~nm}$ (bottom).
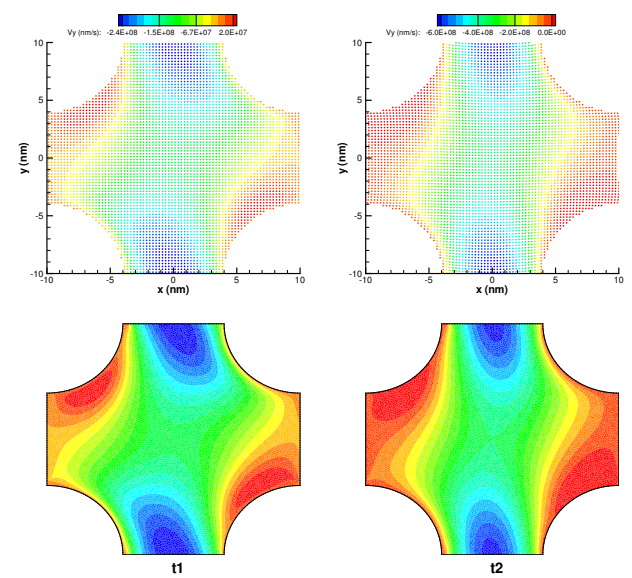

Figure 6: Contour of $v_{y}$ at times $t_{1}=0.264 \mathrm{ps}$ and $t_{2}=2.64 \mathrm{ps}$, computed by $\mathrm{I}^{2} \mathrm{SPH}$ with the spatial resolution $\Delta x=0.25 \mathrm{~nm}($ top $)$ and FEM with $\Delta x=0.2 \mathrm{~nm}$ (bottom). 

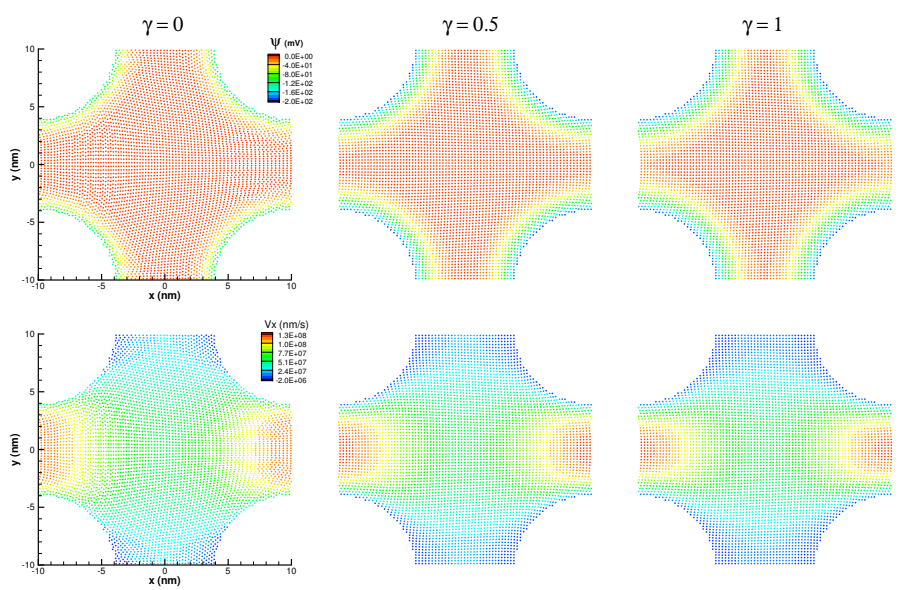

Figure 7: Contour of electrostatic potential (top) and steady-state velocity $v_{x}$ (bottom) distribution in the bulk fluid for different values of $\gamma$, computed by $\mathrm{I}^{2} \mathrm{SPH}$ at $\psi_{0}=-200 \mathrm{mV}$ with the spatial resolution $\Delta x=0.25 \mathrm{~nm}$.

conditions are assumed at the remaining boundaries. The resulting flow velocity is numerically computed, and its steady-state result is compared with the analytical solution in Figure 8 It is evident that the relative $L_{2}$ error decreases linearly with increasing resolution. Note that the second-order convergence was observed in Figures 1 and 2 for the Dirichlet boundary conditions. This indicates that while the discretization of spatial derivatives is second order, the CBF approximation for the Robin boundary condition is first-order accurate.

Next, we consider "patterned" plates, i.e., plates with space-dependent slip lengths and charge potentials, specified as:

$$
\psi_{0}= \begin{cases}\psi_{0,1} & y=H \text { or }-H, x \in\left(-L,-\frac{L}{2}\right) \cup\left(\frac{L}{2}, L\right) \\ \psi_{0,2} \quad y=H \text { or }-H, x \in\left[-\frac{L}{2}, \frac{L}{2}\right]\end{cases}
$$

and

$$
L_{s}= \begin{cases}L_{s, 1} & y=H \text { or }-H, x \in\left(-L,-\frac{L}{2}\right) \cup\left(\frac{L}{2}, L\right) \\ L_{s, 2} & y=H \text { or }-H, x \in\left[-\frac{L}{2}, \frac{L}{2}\right]\end{cases}
$$

with $L=20 \mathrm{~nm}$. The flow then is driven by the applied electric field with $E_{\infty}=$ 

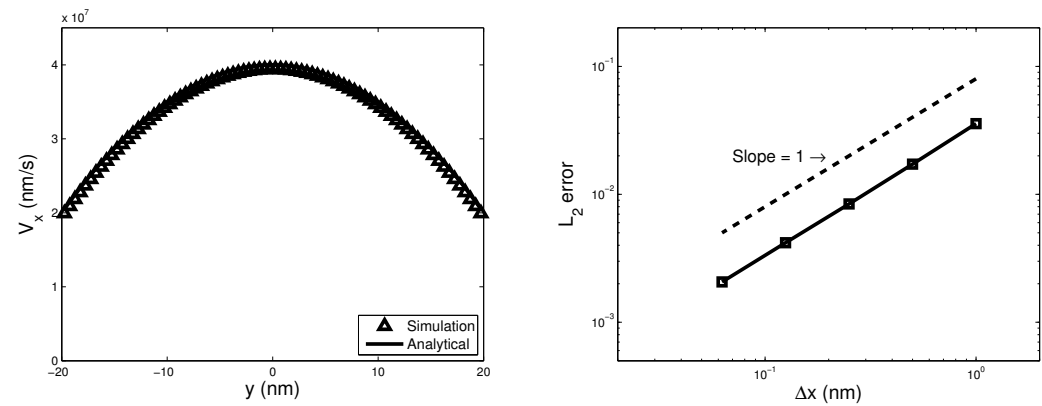

Figure 8: (Left) Steady-state velocity profile of flow subject to Navier slip boundary condition (Eq. 16) with $L_{s}=10 \mathrm{~nm}$ at spatial resolution $\Delta x=0.5 \mathrm{~nm}$, compared with the analytical solution; (right) corresponding $L_{2}$ relative errors at varying spatial resolutions.

$10^{7} \mathrm{~V} / \mathrm{m}$ in the absence of body force $\mathbf{g}$. The resulting electroosmotic flows show very rich behaviors over such designed anisotropic surfaces. As illustrated in Figure 9, the anisotropic surface charge induces convective vortexes in the flow field, similar to the findings of $[63,64$ that the electrokinetic flow depends on the surface charge distribution - not only its total charge. The flow morphology can be further controlled by the slip length in the hydrophobic region. Increasing slip length initially leads to the appearance of additional convective patterns then to a transition to a flow reversal. All of these findings from our $\mathrm{I}^{2} \mathrm{SPH}$ solutions are consistent with previous theoretical studies [63. Thus, our numerical method provides a useful computational tool for directing the practical design of surfaces for electrokinetic flows in microfluidic devices used in mixing and separation processes.

\subsection{Electrophoresis of a Spherical Colloid}

Fourth, we examine the electrophoresis of an insulating 3D spherical colloid charged with a surface potential immersed in an electrolyte solution. Under an applied electric field, Henry's analytical solution [43] predicts the colloid velocity as:

$$
v_{\mathrm{EP}}=E_{\infty} \frac{\epsilon_{r} \epsilon_{0} \psi_{0}}{\mu} m=E_{\infty} \frac{\epsilon_{r} \epsilon_{0} \psi_{0}}{\mu} \frac{2 f(\kappa R)}{3}
$$


a)

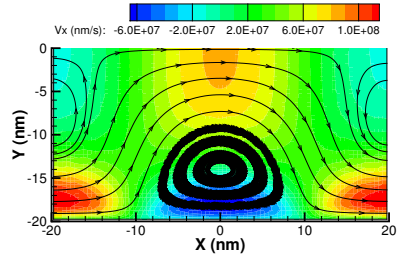

b)

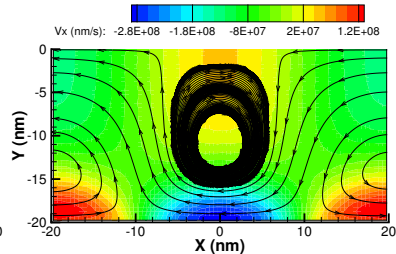

c)

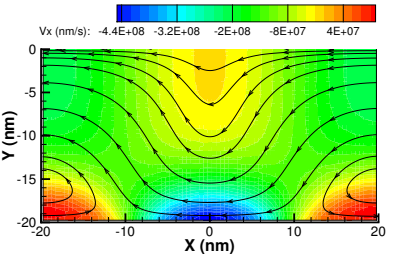

Figure 9: Streamlines of the electroosmotic flow computed at $\lambda_{D}=2 \mathrm{~nm}$ with $\psi_{0,1}=-50 \mathrm{mV}$ and $\psi_{0,2}=40 \mathrm{mV}$ in Eq. (47). From left to right, the local slip length in Eq. 48 is: a) $L_{s, 1}=L_{s, 2}=0$; b) $L_{s, 1}=0.1 \mathrm{~nm}, L_{s, 2}=10 \mathrm{~nm}$; c) $L_{s, 1}=0.1 \mathrm{~nm}, L_{s, 2}=100 \mathrm{~nm}$.

where $R$ is the colloid radius. Here, $f(\kappa R)$ is the Henry's function [43] that depends on the Debye length $\left(\kappa^{-1}=\lambda_{D}\right)$ as:

$f(\kappa R)=1+\frac{1}{16}(\kappa R)^{2}-\frac{5}{48}(\kappa R)^{3}-\frac{1}{8}(\kappa R)^{4}\left[\frac{1}{12}(1-\kappa R)-\left(1-\frac{1}{12}(\kappa R)^{2}\right) e^{\kappa R} E_{1}(\kappa R)\right]$,

where $E_{1}(\kappa R)$ is the exponential integral of order one. This analytical solution is derived by assuming that the electrostatic potential in the fluid can be described by the linearized PB equation and is only valid for weak surface potentials. To validate our numerical method via this analytical solution, we first perform simulations by solving the linearized PB equation:

$$
\nabla^{2} \tilde{\psi}=\kappa^{2} \tilde{\psi} \quad \text { for } \quad \mathbf{x} \in \Omega_{f}
$$

subject to the Dirichlet boundary condition $\psi=\psi_{0}$ on the colloid surface.

The colloid is modeled by freezing a certain number of SPH particles within the volume occupied by the colloid. Those particles are referred to as "solid" particles. Instead of moving the colloid, we fix it in the domain center and drive the fluid flow around it. The no-slip boundary condition is imposed on the colloid surface, and periodic boundary conditions are imposed at the remaining boundaries. The domain size should be sufficiently large to ensure the periodic boundary conditions' effect is negligible, and then the simulations approximate the behavior of a colloid in an infinite domain [65]. 


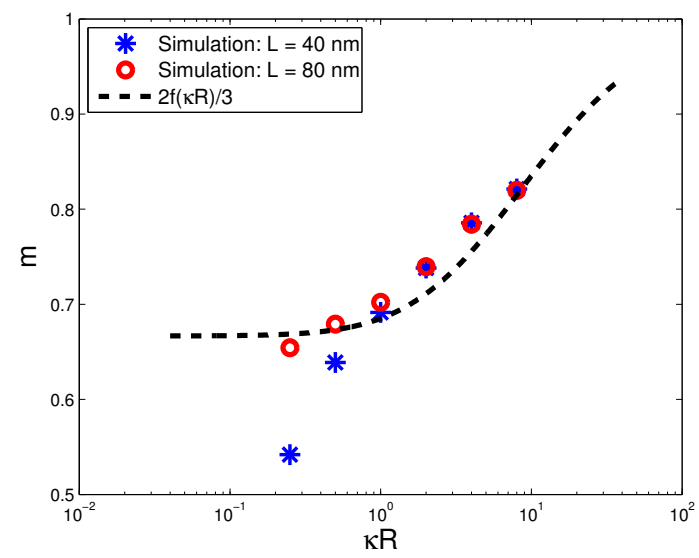

Figure 10: $m=\frac{v_{\mathrm{avg}} \mu}{E_{\infty} \epsilon_{r} \epsilon_{0} \psi_{0}}$ computed by $\mathrm{I}^{2} \mathrm{SPH}$ is compared with $\frac{2 f(\kappa R)}{3}$ at varying values of $\kappa R$.

The applied electric field is computed by numerically solving Eqs. (8) and 10. The average streaming velocity $v_{\text {avg }}$ is calculated from:

$$
v_{\text {avg }}=\frac{1}{V} \int_{V}<v_{x}(x, y, z)>\mathrm{d} x \mathrm{~d} y \mathrm{~d} z
$$

where $\left\langle v_{x}(x, y, z)>\right.$ is the local time average fluid-particle velocity along $\mathbf{e}_{x}$ and $V$ is the fluid domain volume. The computed $v_{\text {avg }}$ by $\mathrm{I}^{2} \mathrm{SPH}$ at different domain sizes are compared with those predicted analytically by Eq. 49p for different values of $\kappa R$. In Figure 10, we see good agreement between $\mathrm{I}^{2} \mathrm{SPH}$ solutions and analytical solutions when the domain size is sufficiently large relative to both the colloid radius and Debye length. Specifically, for a colloid with radius $R=4 \mathrm{~nm}$, the numerical solutions agree well with analytical solutions for the domain with $L=80 \mathrm{~nm}$ for all Debye lengths considered. Meanwhile, for the domain with $L=40 \mathrm{~nm}$, good agreement is found only when the Debye layer is sufficiently thin $(\kappa R \geq 1)$.

Beyond the limit of low surface potential, the Henry's solution is not valid, and the colloid?s electrophoretic mobility usually must be computed numerically. For that and also the effect of ion size, we numerically solve the MPB equation at a high voltage $\left(\psi_{0}=-200 \mathrm{mV}\right)$. The colloid's corresponding elec- 


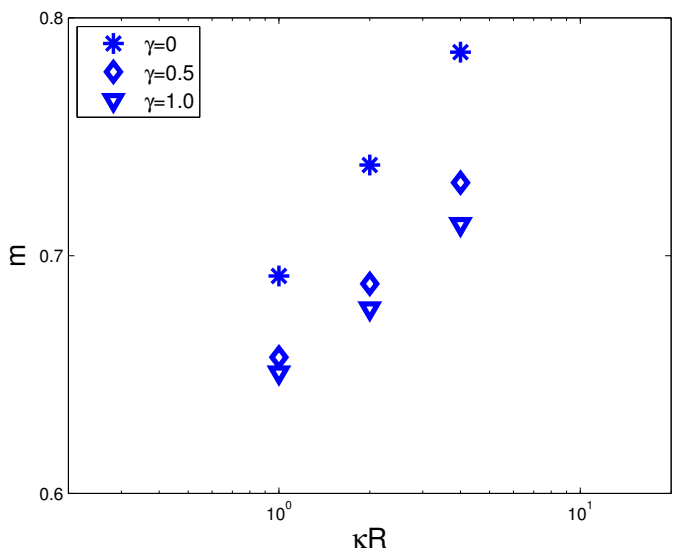

Figure 11: $m=\frac{v_{\mathrm{avg}} \mu}{E_{\infty} \epsilon_{r} \epsilon_{0} \psi_{0}}$ computed by $\mathrm{I}^{2} \mathrm{SPH}$ at varying values of $\kappa R$ and $\gamma$.

trophoretic mobility then is predicted as a function of $\kappa R$ and $\gamma$. With increasing ion size (modeled by increasing $\gamma$ ), the colloid's electrophoretic mobility is found to decrease as depicted in Figure 11. This dependence becomes more pronounced when $\kappa$ increases, or the Debye layer becomes thinner.

\subsection{Electroosmotic Flow through Charged Porous Media}

In our fifth examination, we use the $\mathrm{I}^{2} \mathrm{SPH}$ method for modeling electroosmotic flows through 3D, charged, undeformable synthetic porous media with different porosities. In that, a porous structure is generated as a random packed bed of impermeable spheres with various sizes using the particle-packing method [66]. The porous medium is saturated with electrolyte solution. The applied electric field is numerically computed from Eqs. (8) and (10) with $\lambda_{D}=1 \mathrm{~nm}$ and $\sigma_{s}=0$ for the insulating spheres. A constant charge potential $\left(\psi_{0}=-50 \mathrm{mV}\right)$ is applied on each sphere. The no-slip boundary condition for the fluid velocity is imposed at the surface of spheres, and the periodic boundary conditions at the remaining boundaries. Initially, both the electrostatic potential and fluid velocity are set at zero.

Figure 12 illustrates the distribution of steady-state electroosmotic flow velocity in a porous medium with porosity $\eta=0.67$. As in the previous section, 


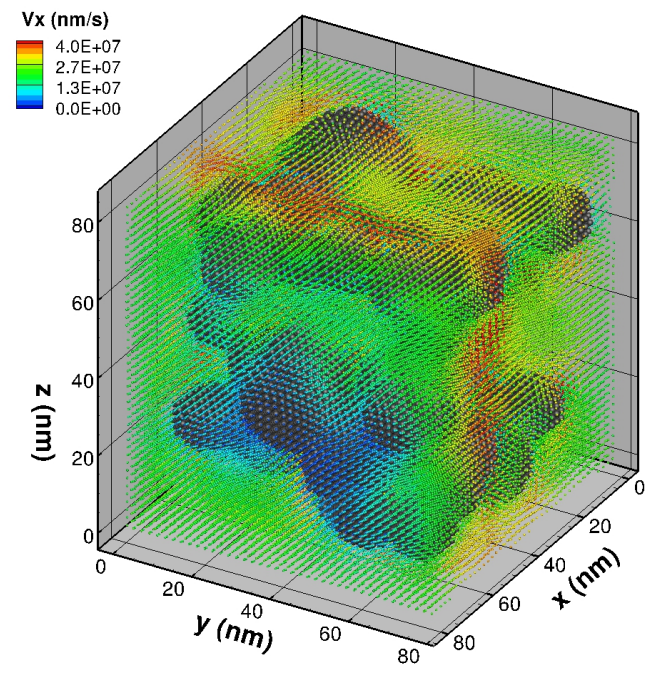

Figure 12: Contour of distribution of electroosmotic flow velocity in a porous medium with porosity $\eta=0.67$.

the average flow velocity $v_{\text {avg }}$ in the porous medium is determined from Eq. 50, where $V$ denotes the total void volume of the porous medium. We first examined the dependence of computed average flow velocity on the strengths of applied electrical field $\left(E_{\infty}\right)$ at two different porosities, which is expected to be linear as shown in Figure 13 .

The presence of non-conducting spheres in electrolytes causes a drop in conductivity relative to that of the bulk solution. It results from the reduction of total void space, a decrease of local electrical field strength, and an increase in migration distance for ions due to the tortuous nature of the intersphere channels. For the flow through charged porous media, composed of poly-sized spheres, the following empirical scaling law has been obtained from experiments for porosity larger than 0.5 [67]:

$$
\frac{v_{\text {sup }}}{E_{\infty}} \propto \eta^{1.5}
$$

where $v_{\text {sup }}$ is the superficial velocity of flow though a porous medium, defined as $v_{\text {sup }}=\eta v_{\text {avg }}$. In Figure 14 , we can see good agreement between the computed 


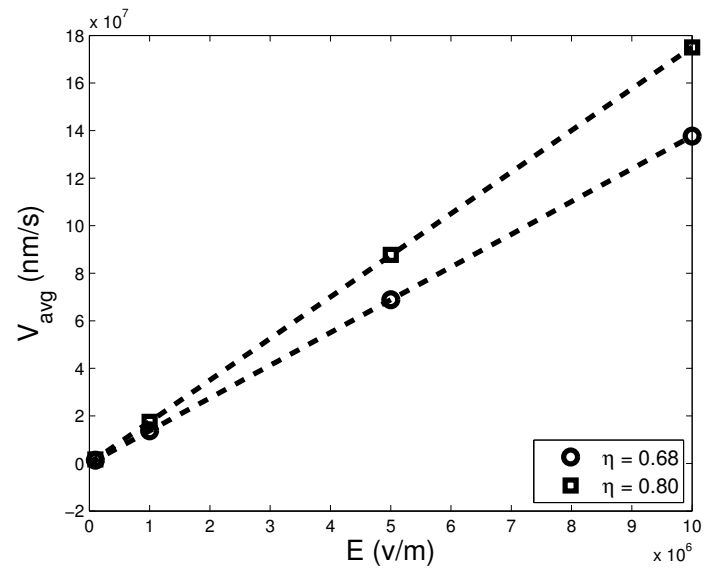

Figure 13: Dependence of average flow velocity $\left(v_{\mathrm{avg}}\right)$ on the strengths of applied electrical field $\left(E_{\infty}\right)$ for different porous media.

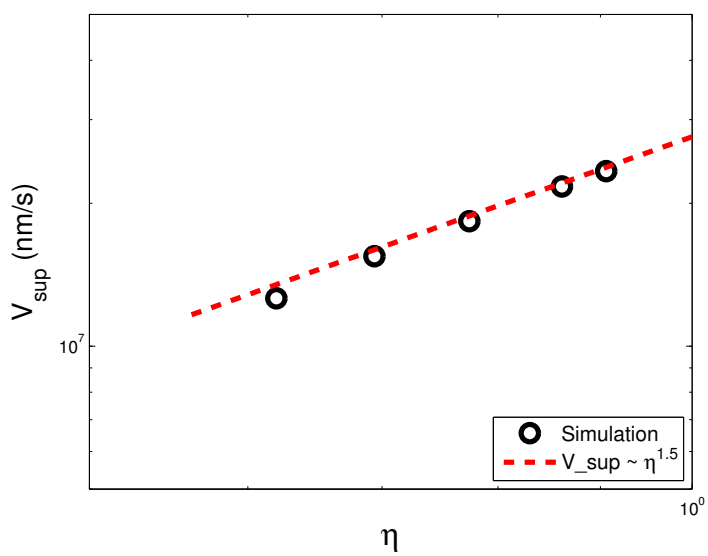

Figure 14: Dependence of the superficial velocity $\left(v_{\text {sup }}\right)$ on porosity $(\eta)$ for electroosmotic flows through $3 \mathrm{D}$ charged porous media. 
$v_{\text {sup }}$ at $E_{\infty}=10^{6} \mathrm{~V} / \mathrm{m}$ and that predicted by Eq. (51). Our simulations predict the same scaling as Eq. 51) with a relative error of $7.9 \%$.

\subsection{Diffusive Mixing in Heterogeneous Microchannel}

Finally, we investigate diffusive mixing of solute in microfluidics by solving the coupled $\mathrm{AD}, \mathrm{NS}$ and $\mathrm{PB}$ equations. In Section 4.3 , we have shown that anisotropic charges and slip lengths on microchannel walls can induce a rich flow behavior with regions of localized flow circulation. Therefore, the same strategy can be used as a part of a microchannel mixer design for enhancing the efficiency of species mixing via advection. In the simulations, we prescribe the heterogeneous charge and slip length on the two microchannel walls as:

$$
\psi_{0}= \begin{cases}\psi_{0,1} & y=-H, x \in \Gamma_{1}=\left[-\frac{2 L}{3},-\frac{L}{3}\right) \cup\left[0, \frac{L}{3}\right) \cup\left[\frac{2 L}{3}, L\right) \\ \psi_{0,2} & y=-H, x \notin \Gamma_{1} \\ \psi_{0,1} & y=H, x \notin \Gamma_{1} \\ \psi_{0,2} & y=H, x \in \Gamma_{1}\end{cases}
$$

and

$$
L_{s}= \begin{cases}L_{s, 1} & y=-H, x \in \Gamma_{1} \\ L_{s, 2} & y=-H, x \notin \Gamma_{1} \\ L_{s, 1} & y=H, x \notin \Gamma_{1} \\ L_{s, 2} & y=H, x \in \Gamma_{1}\end{cases}
$$

with $H=20 \mathrm{~nm}$ and $L=60 \mathrm{~nm}$. Figure 15 shows the electrostatic potential and velocity distribution of the resulting electroosmotic flow with $\lambda_{D}=2 \mathrm{~nm}$ and $E_{\infty}=10^{7} \mathrm{~V} / \mathrm{m}$. The flow pushes the combined stream pattern up and down in the mixing channel, causing extensive folding and stretching of material lines and local circulations.

In the $\mathrm{AD}$ equation (Eq. (25)), the diffusion coefficient is set to $D=5 \times$ $10^{-10} \mathrm{~m}^{2} / \mathrm{s}$, and the equation is solved subject to the boundary conditions as 


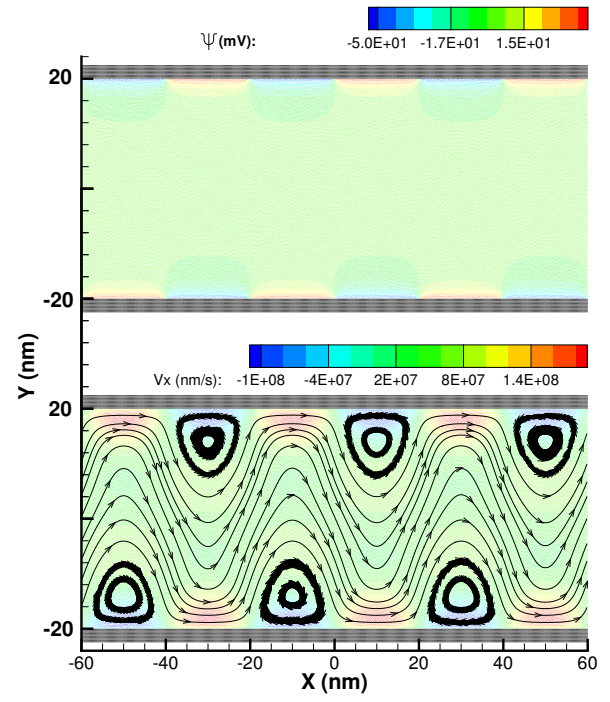

Figure 15: Contour of electrostatic potential (top) and steady-state $v_{x}$ (bottom) distribution in a microchannel with $\psi_{0,1}=-50 \mathrm{mV} ; \psi_{0,2}=40 \mathrm{mV} ; L_{s, 1}=0.1 \mathrm{~nm} ;$ and $L_{s, 2}=100 \mathrm{~nm}$ in Eqs. 52 and 53 .

specified in Eq. 26 with

$$
S_{0}\left(\mathbf{x}_{0}\right)=\left\{\begin{array}{lll}
1 & \text { for } \quad x=-L, y \in\left[-\frac{3 H}{20}, \frac{3 H}{20}\right] \\
0 & \text { for } \quad x=-L, y \in\left(-H,-\frac{3 H}{20}\right) \cup\left(\frac{3 H}{20}, H\right),
\end{array}\right.
$$

i.e., the solute is injected through the central part of the left vertical boundary of the channel. The initial concentration in the channel is set to zero. For comparison, we model mixing in both channels with homogeneous and heterogeneous walls. As illustrated in Figure 16, the mixing efficiency is significantly enhanced in the "heterogeneous" microchannel compared with the "homogeneous" channel. Specifically, in the heterogeneous channel, the solute is better mixed near the channel entrance compared with the homogeneous channel. Previous experimental and numerical studies have used heterogeneous charges on the channel walls to induce circulating flow 68, and enhance mixing efficiency 64]. Here, we demonstrate that heterogeneous slip length provides an additional option in designing optimal microfluidic mixers. 


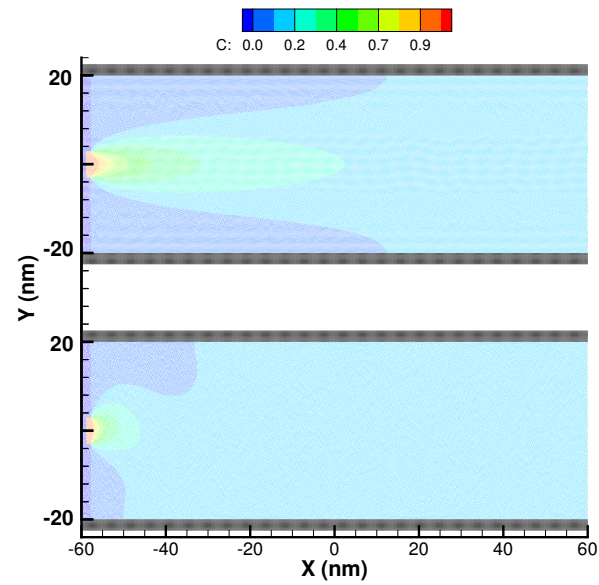

Figure 16: Contour of steady-state solute concentration distribution in a homogeneous microchannel (top) with $\psi_{0,1}=\psi_{0,2}=-50 \mathrm{mV}$ and $L_{s, 1}=L_{s, 2}=0.1 \mathrm{~nm}$ and in a heterogeneous microchannel (bottom) with $\psi_{0,1}=-50 \mathrm{mV} ; \psi_{0,2}=40 \mathrm{mV} ; L_{s, 1}=0.1 \mathrm{~nm}$; and $L_{s, 2}=100$ $\mathrm{nm}$ in Eqs. 52 and 53 .

\section{Conclusion}

We have presented a consistent implicit incompressible SPH discretization of coupled Navier-Stokes, Poisson-Boltzmann, and advection-diffusion equations and demonstrated its accuracy for modeling electrokinetic flows in varying geometries and under various conditions. We also demonstrated that the scheme produced second-order accurate solutions for the coupled PB and NS equations subject to Dirichlet boundary conditions. We obtained a first-order accuracy for these equations subject to the Robin boundary condition using the continuous boundary force method.

We have used the $\mathrm{I}^{2} \mathrm{SPH}$ method to study electroosmotic flows in microchannels and porous media, as well as the electrophoresis of a spherical colloid. For surfaces charged with a high voltage, the ion-size effect also was taken into account via the MPB equation. The simulation results agree very well with the analytical, FEM solutions, and empirical laws. At high charge potentials, the model predicts the electroosmosis and electrophoresis to be significantly affected 
by the ion size.

Finally, we investigated solute transport in electroosmotic flows in a microchannel and demonstrated that a combination of varying slip lengths and surface charges might enhance solute mixing. In $\mathrm{SPH}$, solute is advected by particles, and there is no numerical dissipation for the advection. The current implementation within the highly scalable LAMMPS/Trilinos framework will allow for future extensive explorative studies in large-scale, complicated microfluidic designs for efficient mixing or separation techniques.

Furthermore, due to its Lagrangian nature, the $\mathrm{I}^{2} \mathrm{SPH}$ method can be extended to model complex physical systems, such as moving charged colloids and flexible polymers and proteins with charging blocks immersed in fluids subject to electrokinetic flows.

\section{Acknowledgments}

This work was supported by the Applied Mathematics Program within the U.S. Department of Energy's (DOE) Office of Advanced Scientific Computing Research as part of the Collaboratory on Mathematics for Mesoscopic Modeling of Materials (CM4). This research also used resources of the National Energy Research Scientific Computing Center, a DOE Office of Science User Facility supported by Office of Science under Contract No. DE-AC02-05CH11231. Pacific Northwest National Laboratory is operated by Battelle for DOE under Contract DE-AC05-76RL01830. Sandia National Laboratories is a multi-program laboratory managed and operated by Sandia Corporation, a wholly owned sub-

sidiary of Lockheed Martin Corporation, for the U.S. Department of Energy's National Nuclear Security Administration under contract DE-AC04-94AL85000.

\section{References}

[1] J. J. Monaghan, Smoothed particle hydrodynamics, Reports on Progress in Physics 68 (2005) 1703-1759. 
[2] J. Monaghan, Smoothed particle hydrodynamics and its diverse applications, Annual Review of Fluid Mechanics 44 (2012) 323-346.

[3] X. Hu, N. Adams, A multi-phase SPH method for macroscopic and mesoscopic flows, J. Comput. Phys. 213 (2006) 844-861.

[4] S. Adami, X. Hu, N. Adams, A new surface-tension formulation for multiphase SPH using a reproducing divergence approximation, J. Comput. Phys. 229 (2010) 5011-5021.

[5] N. Tofighi, M. Yildiz, Numerical simulation of single droplet dynamics in three-phase flows using ISPH, Computers \& Mathematics with Applications 66 (2013) 525-536.

[6] A. M. Tartakovsky, A. Panchenko, Pairwise force smoothed particle hydrodynamics model for multiphase flow: Surface tension and contact line dynamics, J. Comput. Phys. 305 (2016) 1119-1146.

[7] W. Pan, A. M. Tartakovsky, J. J. Monaghan, Smoothed particle hydrodynamics non-Newtonian model for ice sheet and ice shelf dynamics, J. Comput. Phys. 242 (2013) 828-842.

[8] X. Xua, P. Yu, A multiscale SPH method for simulating transient viscoelastic flows using bead-spring chain model, J. Nonnewton. Fluid Mech. 229 (2016) 27-42.

[9] A. Vázquez-Quesada, M. Ellero, Rheology and microstructure of noncolloidal suspensions under shear studied with smoothed particle hydrodynamics, J. Nonnewton. Fluid Mech. 000 (2016) 1-11.

[10] W. Pan, D. Li, A. M. Tartakovsky, S. Ahzi, M. Khraisheh, M. Khaleel, A new smoothed particle hydrodynamics non-Newtonian model for friction stir welding: Process modeling and simulation of microstructure evolution in a magnesium alloy, Int. J. Plasticity 48 (2013) 189-204. 
[11] A. Tartakovsky, N. Trask, K. Pan, B. Jones, W. Pan, J. Williams, Smoothed particle hydrodynamics and its applications for multiphase flow and reactive transport in porous media, Computational Geosciences (2015) $1-28$.

[12] W. Pan, M. Daily, N. A. Baker, Numerical calculation of proteinligand binding rates through solution of the Smoluchowski equation using smoothed particle hydrodynamics, BMC Biophys. 8 (2015) 7-18.

[13] S. Liu, J. Savage, G. A. Voth, Mesoscale study of proton transport in proton exchange membranes: Role of morphology, J. Phys. Chem. C 119 (4) (2015) $1753-1762$.

[14] N. J. Quinlan, M. Basa, M. Lastiwka, Truncation error in mesh-free particle methods, International Journal for Numerical Methods in Engineering 66 (2006) 2064-2085.

[15] M. Basa, N. J. Quinlan, M. Lastiwka, Robustness and accuracy of SPH formulations for viscous flow, International Journal for Numerical Methods in Fluids 60 (2009) 1127-1148.

[16] R. Fatehi, M. Manzari, Error estimation in smoothed particle hydrodynamics and a new scheme for second derivatives, Computers \& Mathematics with Applications 61 (2011) 482-498.

[17] N. Trask, M. Maxey, K. Kim, M. Perego, M. L. Parks, K. Yang, J. Xu, A scalable consistent second-order SPH solver for unsteady low Reynolds number flows, Computer Methods in Applied Mechanics and Engineering 289 (2015) 155-178.

[18] S. J. Cummins, M. Rudman, An SPH projection method, J. Comput. Phys. 152 (1999) 584-607.

[19] E.-S. Lee, C. Moulinec, R. Xu, D. Violeau, D. Laurence, P. Stansby, Comparisons of weakly compressible and truly incompressible algorithms for the SPH mesh free particle method, J. Comput. Phys. 227 (2008) 8417-8436. 
[20] S. Shao, E. Lo, Incompressible SPH method for simulating newtonian and non-newtonian flows with a free surface, Advances in Water Resources 26 (2003) 787-800.

[21] M. Ellero, M. Serrano, P. E. nol, Incompressible smoothed particle hydrodynamics, Journal of Computational Physics 226 (2) (2007) 1731 - 1752.

[22] R. Xu, P. Stansby, D. Laurence, Accuracy and stability in incompressible SPH (ISPH) based on the projection method and a new approach, J. Comput. Phys. 228 (18) (2009) 6703-6725.

[23] X. Hu, N. Adams, An incompressible multi-phase SPH method, J. Comput. Phys. 227 (2007) 264-278.

[24] M. Asai, A. M. Aly, Y. Sonda, Y. Sakai, Stabilized incompressible SPH method by relaxing the density invariance condition, Journal of Applied Mathematics 2012 (139583) (2012) 1-24.

[25] A. M. Aly, M. Asai, Three-dimensional incompressible smoothed particle hydrodynamics for simulating fluid flows through porous structures, Transport in Porous Media 110 (3) (2015) 483-502.

[26] A. Chaniotis, D. Poulikakos, P. Koumoutsakos, Remeshed smoothed particle hydrodynamics for the simulation of viscous and heat conducting flows, Journal of Computational Physics 182 (1) (2002) 67-90.

[27] D. Ngo-Cong, C.-D. Tran, N. Mai-Duy, T. Tran-Cong, Incompressible smoothed particle hydrodynamics-moving $\{$ IRBFN $\}$ method for viscous flow problems, Engineering Analysis with Boundary Elements 59 (2015) 172-186.

[28] S. Litvinov, M. Ellero, X. Hu, N. Adams, A splitting scheme for highly dissipative smoothed particle dynamics, Journal of Computational Physics $229(15)(2010) 5457-5464$. 
[29] J. Monaghan, Implicit sph drag and dusty gas dynamics, Journal of Computational Physics 138 (2) (1997) 801-820.

[30] X.-J. Fan, R. Tanner, R. Zheng, Smoothed particle hydrodynamics simulation of non-Newtonian moulding flow, Journal of Non-Newtonian Fluid Mechanics 165 (2010) 219-226.

[31] Y.-W. Han, H.-F. Qiang, H. Liu, W.-R. Gao, An enhanced treatment of boundary conditions in implicit smoothed particle hydrodynamics, Acta Mechanica Sinica 30 (1) (2014) 37-49.

[32] P. V. Liedekerke, B. Smeets, T. Odenthal, E. Tijskens, H. Ramon, Solving microscopic flow problems using stokes equations in $\{\mathrm{SPH}\}$, Computer Physics Communications 184 (7) (2013) 1686-1696.

[33] M. Ihmsen, J. Cornelis, B. Solenthaler, C. Horvath, M. Teschner, Implicit incompressible sph, IEEE Transactions on Visualization and Computer Graphics 20 (3) (2014) 426-435.

[34] W. Pan, J. Bao, A. M. Tartakovsky, Smoothed particle hydrodynamics continuous boundary force method for Navier-Stokes equations subject to a Robin boundary condition, J. Comput. Phys. 259 (2014) 242-259.

[35] R. B. M. Schasfoort, S. Schlautmann, J. Hendrikse, A. van den Berg, Fieldeffect flow control for microfabricated fluidic networks, Science 286 (1999) 942-945.

[36] G. M. Whitesides, The origins and the future of microfluidics, Nature 442 (2006) 368-373.

[37] L. Y. Wick, P. A. Mattle, P. Wattiau, H. Harms, Electrokinetic transport of pah-degrading bacteria in model aquifers and soil, Environ. Sci. Technol. 38 (2004) 4596-4602.

[38] M. E. Suss, T. F. Baumann, W. L. Bourcier, C. M. Spadaccini, K. A. Rose, J. G. Santiago, M. Stadermann, Capacitive desalination with flow-through electrodes, Energy Environ. Sci. 5 (2012) 9511-9519. 
[39] M. S. Shadloo, A. Rahmat, M. Yildiz, A smoothed particle hydrodynamics study on the electrohydrodynamic deformation of a droplet suspended in a neutrally buoyant newtonian fluid, Computational Mechanics 52 (2013) 693-707.

[40] A. Rahmat, N. Tofighi, M. Shadloo, M. Yildiz, Numerical simulation of wall bounded and electrically excited Rayleigh-Taylor instability using incompressible smoothed particle hydrodynamics, Colloids and Surfaces A: Physicochemical and Engineering Aspects 460 (2014) 60-70.

[41] M. S. Kilic, M. Z. Bazant, A. Ajdari, Steric effects in the dynamics of electrolytes at large applied voltages. i. double-layer charging, Phys. Rev. E 75 (2007) 021502.

[42] A. A. Kornyshev, Double-layer in ionic liquids: Paradigm change?, J. Phys. Chem. B 111 (2007) 5545-5557.

[43] D. C. Henry, The cataphoresis of suspended particles. Part I. The equation of cataphoresis, Proc. R. Soc. Lond. A 133 (1931) 106-129.

[44] C. L. Navier, Mémoire sur les lois du mouvement des fluides, Mém. Acad. Roy. Sci. 6 (1823) 389-440.

[45] P. A. Thompson, S. M. Troian, A general boundary condition for liquid flow at solid surfaces, Nature 389 (6649) (1997) 360-362.

[46] L. Bocquet, J.-L. Barrat, Flow boundary conditions from nano-to microscales, Soft Matter 3 (2007) 685-693.

[47] C. Cottin-Bizonne, B. Cross, A. Steinberger, E. Charlaix, Boundary slip on smooth hydrophobic surfaces: Intrinsic effects and possible artifacts, Phys. Rev. Lett. 94 (5) (2005) 056102.

[48] X. Bian, S. Litvinov, R. Qian, M. Ellero, N. A. Adams, Multiscale modeling of particle in suspension with smoothed dissipative particle dynamics, Physics of Fluids 24 (1) (2012) 012002. 
[49] K. Müller, D. Fedosov, G. Gompper, Smoothed dissipative particle dynamics with angular momentum conservation, J. Comput. Phys. 281 (2) (2015) 301-315.

[50] D. J. Price, Smoothed particle hydrodynamics and magnetohydrodynamics, Journal of Computational Physics 231 (3) (2012) 759-794, special Issue: Computational Plasma Physics.

[51] D. I. Graham, J. P. Hughes, Accuracy of sph viscous flow models, International Journal for Numerical Methods in Fluids 56 (8) (2008) 1261-1269.

[52] H. Takeda, S. M. Miyama, M. Sekiya, Numerical simulation of viscous flow by smoothed particle hydrodynamics, Progress of Theoretical Physics 92 (5) (1994) 939-960.

[53] J. P. Morris, P. J. Fox, Y. Zhu, Modeling low Reynolds number incompressible flows using SPH, J. Comput. Phys. 136 (1) (1997) 214-226.

[54] F. Maciá, M. Antuono, L. Gonzalez, A. Colagrossi, Theoretical analysis of the no-slip boundary condition enforcement in SPH methods, Progress of Theoretical Physics 125 (6) (2011) 1091-1121.

[55] D. W. Holmes, J. R. Williams, P. Tilke, Smooth particle hydrodynamics simulations of low reynolds number flows through porous media, International Journal for Numerical and Analytical Methods in Geomechanics 35 (4) (2011) 419-437.

[56] J. J. Monaghan, Simulating free surface flows with SPH, J. Comput. Phys. 110 (2) (1994) 399-406.

[57] M. Heroux, R. Bartlett, V. Howle, et al., An overview of Trilinos, Tech. Rep. SAND2003-2927, Sandia National Laboratories (2003).

[58] E. Bavier, M. Hoemmen, S. Rajamanickam, H. Thornquist, Amesos2 and Belos: Direct and iterative solvers for large sparse linear systems, Scientific Programming 20 (3) (2012) 241-255. 
[59] M. Gee, C. Siefert, J. Hu, R. Tuminaro, M. Sala, ML 5.0 smoothed aggregation user's guide, Tech. Rep. SAND2006-2649, Sandia National Laboratories (2006).

[60] S. Plimpton, Fast parallel algorithms for short-range molecular dynamics, J. Comput. Phys. 117 (1995) 1-19.

[61] A. A. Yazdi, A. Sadeghi, M. H. Saidi, Electrokinetic mixing at high zeta potentials: Ionic size effects on cross stream diffusion, Journal of Colloid and Interface Science 442 (2015) 8-14.

[62] M. Z. Bazant, M. S. Kilic, B. D. Storey, A. Ajdari, Towards an understanding of induced-charge electrokinetics at large applied voltages in concentrated solutions, Adv. Colloid Interface Sci. 152 (1-2) (2009) 48-88.

[63] A. V. Belyaev, O. I. Vinogradova, Electro-osmosis on anisotropic superhydrophobic surfaces, Phys. Rev. Lett. 107 (2011) 098301.

[64] D. Erickson, D. Li, Influence of surface heterogeneity on electrokinetically driven microfluidic mixing, Langmuir 18 (2002) 1883-1892.

[65] G. L. Dent, Aspects of particle sedimentation in dilute flows at finite Reynolds numbers, PhD thesis (1999) Brown University.

[66] D. Coelho, J.-F. Thovert, P. M. Adler, Geometrical and transport properties of random packings of spheres and aspherical particles, Phys. Rev. E 55 (1997) 1959-1978.

[67] R. E. De la Rue, C. W. Tobias, On the conductivity of dispersions, Journal Electrochemical Society 106 (1959) 827-833.

[68] A. D. Stroock, M. Weck, D. T. Chiu, W. T. S. Huck, P. J. A. Kenis, R. F. Ismagilov, G. M. Whitesides, Patterning electro-osmotic flow with patterned surface charge, Phys. Rev. Lett. 84 (2000) 3314-3317. 